\title{
Corrections to the displacement estimation based on analytic minimization of adaptive regularized cost functions for ultrasound elastography
}

\author{
Bo Peng ${ }^{\mathrm{a}, \mathrm{b}, *}$, Junliang Lai ${ }^{\mathrm{b}}$, Ling Wang ${ }^{\mathrm{b}}$ and Dong C. Liu ${ }^{\mathrm{a}}$ \\ ${ }^{a}$ College of Computer Science, Sichuan University, Chengdu, Sichuan, China \\ ${ }^{\mathrm{b}}$ School of Computer Science, Southwest Petroleum University, Chengdu, Sichuan, China
}

\begin{abstract}
Ultrasound elastography has been widely applied in clinical diagnosis. To produce high-quality elastograms, displacement estimation is important to generate fine displacement map from the original ratio-frequency signals. Traditional displacement estimation methods are based on the local information of signals pair, such as cross-correlation method, phase zero estimation. However, the tissue movement is nonlocal during realistic elasticity process due to the compression coming from the surface. Recently, regularized cost functions have been broadly used in ultrasound elastography. In this paper, we tested the using of analytic minimization of adaptive regularized cost function, a combination of different regularized cost functions, to correct the displacement estimation calculated by cross-correlation method directly or by lateral displacement guidance. We have demonstrated that the proposed method exhibit obvious advantages in terms of imaging quality with higher level$\mathrm{s}$ of elastographic signal-to-noise ratio and elastographic contrast-to-noise ratio in the simulation and phantom experiments respectively.
\end{abstract}

Keywords: Ultrasound elastography, adaptive regularized cost function, analytic minimization, displacement estimation

\section{Introduction}

In ultrasound elastography, a minor force exerted by the probe is required. When pressed on human tissues, the probe will generate small deformations on tissues. Accordingly, an accurate estimation of displacement plays an essential role in strain computation to achieve a high-quality elastogram. Because the digitalized ultrasound echo signals are sampled discretely, the displacement estimation based on similarity matching technique are prone to producing errors. Due to the limited sampling intervals, some proven techniques have been used to reduce these estimated errors. These techniques can be classified into the following types: up-sampling from the original signals [1,2], curve- or parabola-fitting on the original signals $[3,4]$, and curve- or parabola-fitting on the cross-correlation functions [5-7].

\footnotetext{
${ }^{*}$ Corresponding author. Bo Peng, College of Computer Science, Sichuan University, Chengdu, Sichuan, China. Tel.:02883037528; Fax:028-83037528; E-mail:pengbo.cs@gmail.com.
}

0959-2989/14/\$27.50 @ 2014 - IOS Press and the authors. 
It should be noted that curve- or parabola-fitting on the cross-correlation function has already replaced the aforementioned two methods, and has been broadly applied in the sub-sample displacement estimations. In ultrasound elastography, curve-fitting techniques are generally used for the estimation of sub-sample displacement along one direction, such as the parabola-fitting [6], cosine-fitting [7], and spline-fitting [2] under one-dimensional (1D) conditions , or for the multi-dimension polynomial fitting displacement estimation [8]. In spite of its efficient computation and favorable precision, the calculated sub-sample displacement by curve fitting is often the optimal solution of the function composed of several cross-correlation coefficients. However, the displacement estimation results are not accurate under several circumstances. For example, the noises mixed in the echo signals can affect the locally optimal solution. Moreover, the forced movements of human tissues are often continuous, and this result in the imprecise estimation by curve fitting. Based on the hypothesis of displacement continuity, Rivaz et al. have introduced a real-time elastography technique using analytic minimization (AM) of regularized cost functions [9]. The sample point displacement estimation was then converted to accurate sub-sample displacement estimation, and the obtained displacement field was continuous and smooth. However, the displacement field is more likely to be over-smoothed using AM of regularized cost functions, especially in the boundary regions connecting different tissues. So the tissues boundaries cannot be distinguished easily and accurately.

In this paper, we present a displacement correction method based on analytic minimization (AM) of adaptive cost functions for reconstructing elastograms. This method gives precise sub-sample axial displacements by providing flexible cost function choices (i.e., cost function is not fixed). Simulation and experimental results are provided for quantitative validation.

\section{Materials and method}

\subsection{Initial displacement calculations based on quality measurement}

The displacement estimation methods based on cross-correlation analysis can be applied properly in only one direction or the combination of the two. To ensure an accurate initial displacement estimation of seed RF-line, the propagation of estimation errors to the adjacent points along RF-line should be avoided whenever possible, and therefore the multi-directional guidance tracking method [10] is used. Furthermore, lateral guidance is a common method for displacement initialization. In this paper, initial displacements of non-seed RF-lines are assigned by using lateral guidance. Although the calculations of the initial displacements of all non-seed lines can be omitted, the errors in the lateral displacement calculations may be amplified, imposing some bad effects on the quality of image. Accordingly, multiple seed RF-lines and quality measurement are both necessary. When performing the initial displacement estimations on the sample points along the non-seed RF lines, the displacements of the adjacent locations along RF-line are evaluated by the correlation-based quality indicator [11], since the lateral displacement is a known quantity. Finally, the sample point whose displacement with the highest cross-correlation coefficient is elected as the initial displacement of current point.

In order to reduce the computational complexity and to avoid worm noise of elastogram, the number of windows to estimate the displacements should be limited. The size and overlapping ratio of the comparison window define the number of the cross-correlation windows in the calculations of RF-lines before and after compression. Accordingly, we cannot obtain the displacements of all sample points along RF-lines. Aiming at matching to the following design of regularized cost function, some simple prepro- 
cessing should be made, thus, making each sample point in the RF-line matched to the displacement of the corresponding cross-correlation window.

\subsection{Analytic minimization (AM) of adaptive regularized cost functions}

The elastogram of phantoms exhibit a noisy appearance, especially in boundary region between inclusion and background if the medium contains complex structure. At least two factors contribute to the strong noise in boundary region. First, while the elasticity phantom we have used is compressible and locally heterogeneous in boundary region, some complicated local variability may occur. Second, displacement is estimated by classical 1-D time delay estimation, because biological tissues do not move in only one dimension when compressed, and displacement estimation is easy to be effected by decorrelation errors. Adjacent RF-lines are usually processed independently in displacement calculation.

In the AM of single regularized cost functions, displacement of all samples along a RF-line can be regularized in the same way. However, over or less smoothness may appear in the boundary region, leading to an ambiguous boundary in elastogram. To reduce the noise in boundary region, we will perform corrections to the displacements of difference regions with corresponding regularized cost functions. The regularized cost functions proposed in this study meets two criteria: 1) smoothing on the displacement is reduced or unchanged in the boundary region, and 2) smoothing on the displacement is enhanced in inclusion and background areas. The regularized cost functions proposed by Rivaz, et al. $[9,12]$ can meet the first criterion, and the elastogram should be further improved by enhancing displacement smooth.

(1) Regularized cost functions for displacement smoothness (also called as the standard 1D regularized cost functions): with regard to the displacement consistency constraint, we should consider the displacement consistency of the current sample with adjacent samples along the axial direction and the lateral direction, with the expression described as:

$$
E_{c}\left(\Delta a_{1}, \ldots \Delta a_{m}\right)=\sum_{i=1}^{m}\left\{\alpha\left(a_{i}+\Delta a_{i}-a_{i-1}-\Delta a_{i-1}\right)^{2}+\beta\left(a_{i}+\Delta a_{i}-d_{i, j-1}\right)^{2}\right\}
$$

(2) Regularized cost functions for enhancing the displacement smoothness (also called as the smoothed 1D regularized cost function): to improve the smoothing effects, we should consider the displacement consistency of the sample point with two adjacent samples along the axial direction and one sample along the lateral direction, with the expression described as:

$$
\begin{aligned}
E_{c}\left(\Delta a_{1}, \ldots \Delta a_{m}\right) & =\sum_{i=1}^{m}\left\{\alpha\left[\left(a_{i}+\Delta a_{i}-a_{i-1}-\Delta a_{i-1}\right)^{2}+\left(a_{i}+\Delta a_{i}-a_{i-2}-\Delta a_{i-2}\right)^{2}\right]\right. \\
& \left.+\beta\left(a_{i}+\Delta a_{i}-d_{i, j-1}\right)^{2}\right\}
\end{aligned}
$$

in which $\Delta a_{1}, \ldots \Delta a_{m}$ all denote the required offsets of displacement; $a_{i}$ is the initial displacement calculated by cross-correlation algorithm; $d_{i, j-1}=a_{i, j-1}+\Delta a_{i, j-1}$ denotes the displacements of the samples in the left location, $\alpha$ and $\beta$ are the adjustable constraint factors for axial and lateral displacements, respectively. According to the first-order Taylor formula, the expansion of $\mathrm{I}_{2}\left(i+a_{i}+\Delta a_{i}, j\right)$ with regard 
to $a_{i}$ can be approximately written as:

$$
\begin{aligned}
C_{j}\left(\Delta a_{1}, \ldots \Delta a_{m}\right) & =\sum_{i=1}^{m}\left\{\left[\mathrm{I}_{1}(i, j)-\mathrm{I}_{2}\left(i+a_{i}+\Delta a_{i}, j\right)-\mathrm{I}_{2}^{\prime}{ }^{2}\left(i+a_{i}, j\right) \Delta a_{i}\right]^{2}\right. \\
& +\alpha\left[\left(a_{i}+\Delta a_{i}-a_{i-1}-\Delta a_{i-1}\right)^{2}+\left(a_{i}+\Delta a_{i}-a_{i-2}-\Delta a_{i-2}\right)^{2}\right] \\
& \left.+\beta\left(a_{i}+\Delta a_{i}-d_{i, j-1}\right)^{2}\right\}
\end{aligned}
$$

in which $\mathrm{I}_{2}^{\prime}$ is the derivative of the $\mathrm{I}_{2}^{\prime}{ }_{2}^{2}\left(i+a_{i}, j\right)$ in the axial direction. The optimum $\Delta a_{i}$ can be obtained when the partial derivation of $C_{j}$ with respect to $\Delta a_{i}$ is zero, i.e., and the problem $\partial C_{j} / \partial \Delta a_{i}=0$ in Eq. (3) can be changed to solving system of linear equations,

$$
\begin{aligned}
& \left(\mathrm{I}^{\prime}{ }^{2}+\alpha D_{2}+\beta \hat{\mathrm{I}}\right) \Delta a_{j}=\mathrm{I}_{2}^{\prime} e-\left(D_{2}+\beta \hat{\mathrm{I}}\right) a_{j}+\beta a_{j-1} \\
& D_{2}=\left[\begin{array}{cccccccc}
2 & -1 & -1 & 0 & 0 & 0 & \cdots & 0 \\
-1 & 3 & -1 & -1 & 0 & 0 & \cdots & 0 \\
-1 & -1 & 4 & -1 & -1 & 0 & \cdots & 0 \\
0 & -1 & -1 & 4 & -1 & -1 & \ddots & 0 \\
0 & \ddots & \ddots & \ddots & \ddots & \ddots & \ddots & 0 \\
0 & 0 & 0 & -1 & -1 & 4 & -1 & -1 \\
\vdots & \ddots & \ddots & 0 & -1 & -1 & 3 & -1 \\
0 & \cdots & 0 & 0 & 0 & -1 & -1 & 2
\end{array}\right] \text {. }
\end{aligned}
$$

The formula in AM of the standard 1D regularized cost functions is similar with Eq. (4), in which

$$
D_{1}=\left[\begin{array}{cccccc}
1 & -1 & 0 & 0 & \cdots & 0 \\
-1 & 2 & -1 & 0 & \cdots & 0 \\
0 & -1 & 2 & -1 & \cdots & 0 \\
\vdots & & & & \ddots \\
0 & 0 & \cdots & 0 & -1 & 1
\end{array}\right]
$$

Furthermore, in this case, simple symbol substitutions are performed for Eq. (4), $\mathrm{y}=\mathrm{I}_{2}^{\prime}{ }_{2} e-\left(D_{2}+\right.$ $\beta \hat{\mathrm{I}}) a_{j}+\beta a_{j-1}, M=\mathrm{I}_{2}^{\prime}{ }_{2}{ }^{2}+\alpha D_{2}+\beta \hat{\mathrm{I}}, x=\Delta a_{j}$ where $x$ and $y$ are column vectors, $M$ is a matrix. In the case of a discrete linear inverse problem in describing a linear system, the problem can be written as:

$$
x=M^{-1} y
$$

$$
y=M x .
$$

The objective of an inverse problem is to find the best model x (i.e. the optimal $\Delta a_{i}$ values). Accordingly, the forward problem relates the optimal $\Delta a_{i}$ values to the data that we obtained from original RF signals and initial integer displacement. 
In the calculations of sub-sample displacement, the coefficient matrix $M$ is important for seeking the optimal $x$. This is because the regularization weight $\alpha$ and $\beta$ are tunable parameters. The two weights penalize the difference of axial displacement between the each sample and its neighbor samples. Usually, they are positive, large weights are applied for prevent regions with high local decorrelation but makes the share of the data term in the cost function very small and causes over-smoothing in axial direction or lateral direction. Due to the limit to $\alpha$ and $\beta$, the coefficient matrix $M$ is strictly diagonally dominant (i.e. $\left|M_{i i}\right|>\sum_{j \neq i}\left|M_{i j}\right|$ for all $i$ where $M_{i j}$ is the $i, j$ th element of $M$, whatever single cost function or the combination of them we used ), symmetric and all diagonal entries are positive. Therefore, it is positive definite, which means that setting the gradient of $C$ to zero results in the global minimum of $C$ . The 1D AM results presented in this work are obtained with one iteration of the above equation.

\subsection{Selection of adaptive regularized cost functions}

The elastogram can reflect the texture and structure of human tissues. On the basis of the rough strain information estimated from initial displacement, it is easy to make a judgement on whether the existing estimation point locates in the boundary region, or in the inclusion and background region.

Suppose the initial axial displacement of the current sample is $a_{i, j}$, the corresponding initial axial displacements of the neighbouring samples are $a_{i-1, j}$ and $a_{i+1, j}$, respectively; then the corrections to displacement estimation can be achieved by performing adaptive calculations with different regularized cost functions. The regularized cost function can be selected according to the roughly strain information of the current sample $s_{i, j}$ and its neighbor samples $s_{i-1, j}$ and $s_{i+1, j}$.

The regularized cost function can be selected according to the roughly estimated strain of the current sample. Then the corrections to displacement estimation can be achieved by performing adaptive calculations with different regularized cost functions. The expression is similar as Eq. (4), written as:

$$
\left(\mathrm{I}_{2}^{\prime}{ }^{2}+\alpha D+\beta \hat{I}\right) \Delta a_{j}=I_{2}^{\prime} e-(D+\beta \hat{I}) a_{j}+\beta a_{j-1} .
$$

The matrix $D$ is adaptive according to the following description:

$$
D(i,:)= \begin{cases}D_{2}(i,:) & s_{i} \in \text { background } \| s_{i} \in \text { inclusion } \\ D_{1}(i,:) & \text { others }\end{cases}
$$

in which the threshold value is related to the strain in the inclusion and background regions. Moreover, in accordance with the practical requirements in calculations, the local strain estimation region centering on the current sample, can be expanded upward and downward to improve the accuracy of adaptive calculations.

\section{Simulations and experiments}

\subsection{Simulation methods}

The ultrasonic echo data are simulated by a simple linear system method $[13,14]$. In echo simulations, the center frequency of the probe was set as $5 \mathrm{MHz}$, with the fractional order of $75 \%$. The sampling 
frequency was $40 \mathrm{MHz}$, and the velocity of sound speed was a constant, $1540 \mathrm{~m} / \mathrm{s}$. A two-dimensional uniform elastic tissue model $(40 * 40 \mathrm{~mm})$ was adopted. To calculate the axial strain, we used a 1D axial strain model (with the assumption of $d x=0$ ), in which each frame was composed of 300 RF-lines, each with 550 sample points. Different amounts of strain were applied on the model, specifically, $0.1 \%, 0.5 \%$, $1 \%, 2 \%, 3 \%$ and $5 \%$. Two models were adopted in the simulations: a uniform model and a non-uniform model. Mainly for performing simulations on a uniform human tissue without any gelosis or cyst, the former model can be used to study the elastographic signal-to-noise ratio (SNRe) [15] for elastography algorithms. The second model is chiefly adopted in the simulations on a non-uniform human tissue containing a tumor or a soft cyst, which can be used to study the elastographic contrast-to-noise ratio (CNRe) [16] for elastography algorithms.

\subsection{Phantom experiments}

The ultrasound imaging system in this study is a iMago C21 ultrasound machine (Saset healthcare Inc., Chengdu, China), and the adopted probe is a linear array probe with 128 arrays, whose center frequency and fractional bandwidth are $5 \mathrm{MHz}$ and $75 \%$, respectively. The phantom experiments were performed on a customized elasticity phantom CIRS Model 049 (CIRS Inc., Norfolk, Virginia, USA), and the imaging object was a hard inclusion of $10-\mathrm{mm}$ diameter, with the elasticity modulus of $63 \mathrm{kPa}$. The RF sampling frequency was $40 \mathrm{MHz}$. During the data-acquiring process, the probe was pressed or released manually in the axial direction at a constant speed without any additional control device. 100 frames of echo sequences were collected in the compression. The phantom experiments were then conducted by scanning RF echo signals in real time.

\subsection{Implementation of the methods}

The following three methods were performed for comparisons in Matlab platform. The initial displacement of seed RF-line was calculated using cross-correlation method based on axial guidance, and the initial displacement of non-seed RF-line was calculated by lateral guidance. The displacements of RF-lines were then corrected by AM of standard 1D regularized cost functions proposed by Rivaz,et al. [12], denoted as CCM_AM.

The initial displacement of seed RF-line was calculated using cross-correlation method based on multidirectional guidance, and the initial displacement of non-seed RF-line was calculated by lateral guidance combined with quality measurement (QM). The displacements of RF-lines were then corrected by AM of standard 1D regularized cost functions, denoted as CCM_QM_AM.

Compared with CCM_QM_AM, the third method corrected the intital displacements by the proposed 1D adaptive regularized cost function, denoted as CCM_QM_SA_AM.

In these implementations, the cross-correlation window for motion tracking included 40 sample points, and the interval was 10 samples with the overlapping ratio of $75 \%$. The size of SG-I differential filter, which is used for strain estimation, was 11 . The axial and lateral constraint factors $\alpha$ and $\beta$ for displacement constraint was 0.5 and 0.005 , respectively.

\section{Experiment and results}

\subsection{Simulation experiment and results}

Figure 1 shows the displacements and corresponding representative elastograms calculated by the three methods. In the simulations, the stain of tissues was $1 \%$, and Gaussian noises were added into the 


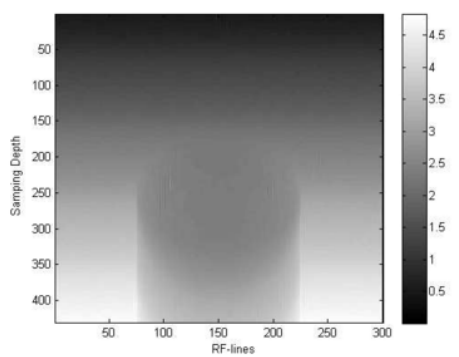

(a)

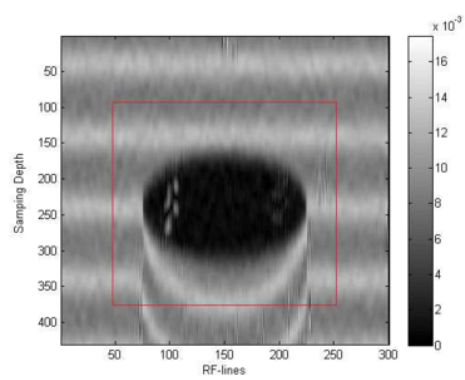

(d)

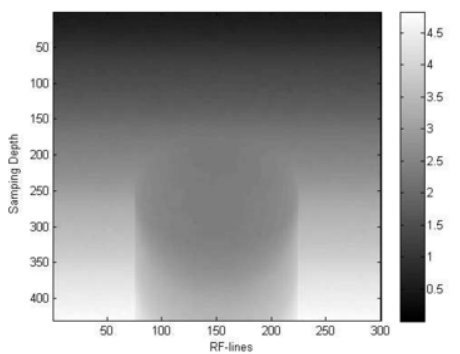

(b)

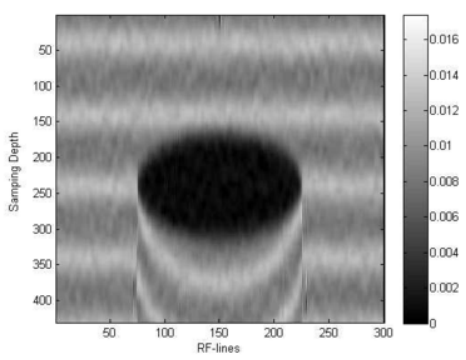

(e)

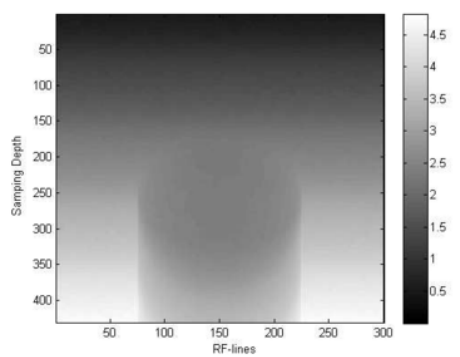

(c)

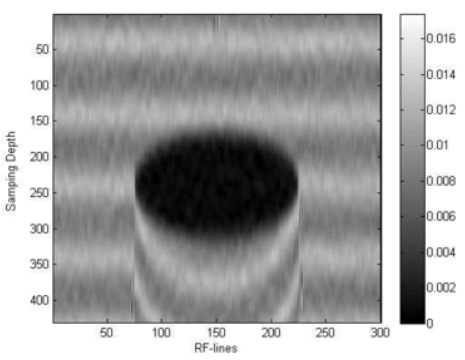

(f)

Fig. 1. Displacement estimation and elastogram quality using the different methods. Displacement map by CCM_AM (a), CCM_QM_AM (b), CCM_QM_SA_AM (c); (d), (e) and (f) represent the elastograms of (a) $(\mathrm{CNRe}=22.3 \overline{6} 37)$, (b) $(\mathrm{CNRe}=24.8355)$, and (c) $(\mathrm{CNRe}=30.2673)$.

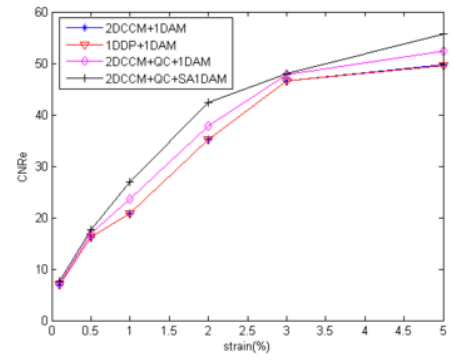

(a)

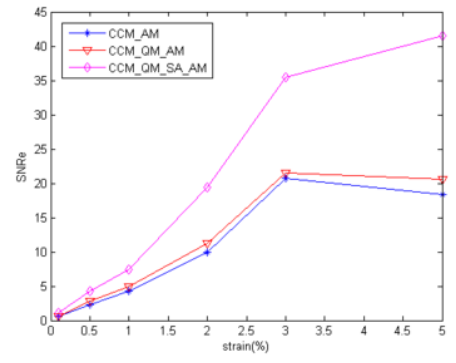

(b)

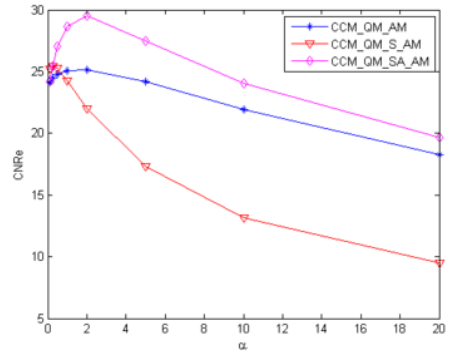

(c)

Fig. 2. Comparisons of elastogram performances (a). CNRe, (b). SNRe, (c). Effects of axial constraint factor on CNRe in elastograms.

specific RF-lines. As shown in Figure 1(a), using the first method, the wrong displacement information was spreaded in both axial and lateral directions and the noises were amplified in strain estimation. In contrast, no displacement estimation is improperly amplified with the use of quality measurement based on guidance displacement, and also no obvious wrong displacement is estimated, as show in Figures 1(b) and $1(\mathrm{c})$.

Comparison of the CNRe in calculations of elasticity imaging by the different methods is shown in Figure 2(a), with a varying strain from $0.01 \%$ to $5 \%$. As stated previously, the phantom in the simulations included a hard circular inclusion. As shown in Figure 2(a), when the strain is below 0.5\%, the CNRes 
calculated by the different methods are of tiny difference. As the strain increased to between $1 \%$ and 5\%, the CNRe calculated by CCM_QM_SA_AM method is obviously higher than CNRes calculated by other methods. The CNRes by CCM_QM_AM method is the second highest, which is superior to the results obtained by the method without quality measurement on guidance displacement. The comparison results indicate that quality measurement is essential when displacement is initialized by lateral guidance, because quality measurement is conductive to the removal of wrong guidance displacement. As shown in Figure 1(d), the circular region was selected as the inclusion region while the regions between the circular and rectangular regions were the background region.

A uniform phantom was used for the SNRe calculations. To obtain more representative and accurate results, the quantitative results were the average values by five separate simulations. The SNRe of each frame of data was $20 \mathrm{~dB}$ with the addition of white Gaussian noises. As shown in Figure 2(b), the calculated SNRes by the different methods are close to each other when the strain varies from $0.1 \%$ to $1 \%$. As the strain increased to be $2 \%-5 \%$, the SNRe calculated by the proposed CCM_QM_SA_AM is apparently higher than the results from other methods. Moreover, the calculated SNRe by CCM_QM_SA_AM presents an increase while the results calculated by the other methods decrease, with the increasing strain from $3 \%$ to $5 \%$. On the whole, using quality measurement strategy, the accuracy of displacement tracking can be enhanced. Most remarkably, the quality of elastogram can be most significantly improved by AM of adaptive regularized cost functions. The region for the calculation of SNRe was the whole elastogram.

The effects of axial constrain factor on the CNRe in elastograms, calculated by these three methods, were compared and presented in Figure 2(c). In these experiments, the axial constraint factor varied from 0.125 to 20 , and the phantom in the simulations contained a hard circular inclusion, with a strain of 1\%. As shown in Figure 2(c), using AM of adaptive regularized cost functions (CCM_QM_SA_AM), an optimal CNRe can be obtained when the axial constraint factor is 2 . With regard to the proposed CCM_QM_SA_AM method, the CNRe of elastogram increases as the axial constraint factor ranges from 0.125 to 0.25 , and then decreases gradually. The CNRe calculated by CCM_QM_AM method decreases when the axial constraint factor exceeds 2. Overall, the calculated CNRe by the three methods all decrease when $\alpha$ varies from 2 to 20. The results indicate that the CNRe of elastogram cannot be improved only by manipulating the axial constraint factor $\alpha$. However, the CNRe of elastogram using CCM_QM_SA_AM can significantly improve the visibility of the marginal area between inclusion and background. This is because we should firstly identify the current estimation location and decide whether it is in inclusion region, background region, or boundary region based on the prior knowledge. Especially for a real-time monitoring treatment, this method exhibit distinct advantages in an accurate identification of an organ's boundary.

\subsection{Phantom experiment and results}

As shown in Figure 3, in the elastogram obtained by the proposed method, we can find a hard inclusion in the scanned elasticity phantom. In the elastogram obtained by CCM_AM, we can also observe a hard inclusion. Unfortunately, the image exhibit a poor quality and obvious noises. The cross-correlation window for motion tracking in real phantom experiments was 100 sample points, and the interval was 20 points, with an overlapping ratio of $75 \%$. The size of the SG-I differential filter was 31 . The axial and lateral constraint factors, $\alpha$ and $\beta$, were 10 and 0.005 , respectively. In AM of adaptive regularized cost functions, the thresholds for the background and inclusion were set as 0.004 and 0.002 , respectively. 


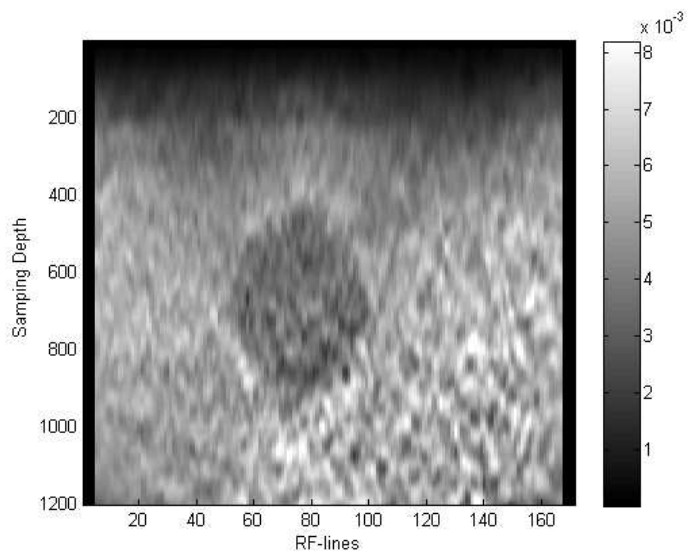

(a)

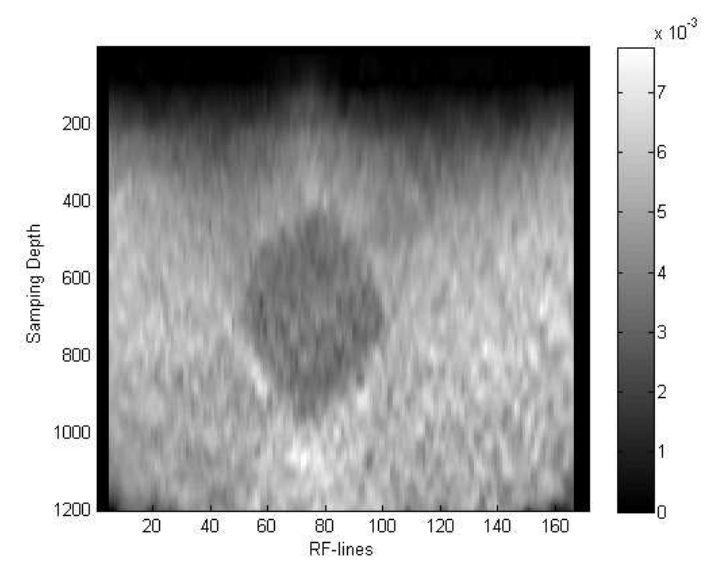

(b)

Fig. 3. Elastography of phantom calculated by CCM_AM (a) and by CCM_QM_SA_AM (b).

\section{Discussion and Conclusion}

A combination of multi-directional guidance tracking method with the quality measurement is applied in the initial displacement of seed RF-line. Such method not only decreases the estimation error and avoids error propagation, but also increase the accuracy for selection of adaptive regularized cost functions. We have shown in Figure 1 that multi-directional guidance tracking is more robust to the signals decorrelation while quality measurement is able to reduce errors in displacement propagation. Furthermore, the computation for more seed RF-lines will achieve more accurate initial displacement estimation. The CNR and SNR results in Figures 2(a) and 2(b) seem to indicate that the adaptive cost function provides a promising performance for elastograms. Especially in Figure 2(a), the proposed adaptive cost function approach creates a smoother elastograms than standard single cost function while the contrast is preserved. In Figure 2(b), the lateral regularized weight $\beta$, in Eq. (8) was kept constant at throughout these experiments. The axial regularized weight was varied from 0.125 to 20 . Figure 2(b) indicates that the maximum CNRe of elastogram cannot be obtained correspondingly by only increasing the axial regularized weight. In addition to that, adaptive cost function is effective to reduce the over-smoothing effect and achieve the maximum CNRe in these experiments while varying the axial regularized weight.

In this study, we have proposed a displacement correction technique using AM of adaptive regularized cost functions. The proposed method reduces the strong noise in boundary region and achieves more accurate sub-sample displacement estimations by substituting adaptive regularized cost functions for solo regularized cost function. The simulation and phantom results indicate that the proposed methods exhibit obvious advantages in terms of imaging quality with higher SNRe and CNRe. Besides, the proposed method does not over-smooth the boundary region between inclusion and background. By comparing the calculated CNRe and SNRe, the superiority of adaptive regularized cost functions is proved. The phantom imaging results indicate that the motion of scanned tissues can be estimated precisely with the use of the proposed method, and the obtained elastography are of high SNR and CNR. Collectively, the proposed method CCM_QM_SA_AM will be of great significances in the developments of realtime elastography and their clinical applications, especially in real-time monitoring for thermal ablation treatments. This new method will be tested on clinical ultrasound imaging and parameter optimizations in the future. 


\section{Acknowledgment}

This work was partially supported by scientific research starting project of SWPU (No.2014QHZ023), the applied basic research programs of science and technology commission Foundation of Sichuan Province (No.2013JY0134).

\section{References}

[1] E. Konofagou and J. Ophir, A new elastographic method for estimation and imaging of lateral displacements, lateral strains, corrected axial strains and Poisson's ratios in tissues, Ultrasound in medicine \& biology 24 (1998), 1183-1199.

[2] F. Viola and W.F.Walker, A spline-based algorithm for continuous time-delay estimation using sampled data, Ultrasonics, Ferroelectrics and Frequency Control, IEEE Transactions on 52 (2005), 80-93.

[3] R. Zahiri-Azar and S.E. Salcudean, Time-delay estimation in ultrasound echo signals using individual sample tracking, Ultrasonics, Ferroelectrics and Frequency Control, IEEE Transactions on 55 (2008), 2640-2650.

[4] F. Viola, R. L. Coe, K. Owen, D. A. Guenther, and W. F. Walker, Multi-dimensional spline-based estimator (MUSE) for motion estimation: algorithm development and initial results, Annals of biomedical engineering 36 (2008), 1942-1960.

[5] B. Geiman, L. Bohs, M. Anderson, S. Breit, and G. Trahey, A comparison of algorithms for tracking sub-pixel speckle motion, Proceedings of IEEE Ultrasonics Symposium, 1997 (1997), 1239-1242.

[6] S.G. Foster, P.M. Embree, and W.D. O’Brien, Flow velocity profile via time-domain correlation: error analysis and computer simulation, Ultrasonics, Ferroelectrics and Frequency Control, IEEE Transactions on 37 (1990), 164-175.

[7] P.G.M. De Jong, T. Arts, A.P.G. Hoeks, and R. S. Reneman, Determination of tissue motion velocity by correlation interpolation of pulsed ultrasonic echo signals, Ultrasonic Imaging 12 (1990), 84-98.

[8] R.Z. Azar, O. Goksel, and S.E. Salcudean, Sub-sample displacement estimation from digitized ultrasound rf signals using multi-dimensional polynomial fitting of the cross-correlation function, Ultrasonics, Ferroelectrics and Frequency Control, IEEE Transactions on 57 (2010), 2403-2420.

[9] H. Rivaz, E. M. Boctor, M. A. Choti, and G. D. Hager, Real-Time Regularized Ultrasound Elastography, Medical Imaging, IEEE Transactions on 30 (2011), 928-945.

[10] S. Cui, C. Peng, and D.C. Liu, Multi-Direction Guidance Motion Tracking for Real-Time Ultrasound Strain Imaging, Proc. Int. Conf. Bioinformatics and Biomedical Engineering (iCBBE), 2010 (2010), 1-4.

[11] L. Chen, G.M. Treece, J.E. Lindop A.H. Gee and R.W. Prager, A quality-guided displacement tracking algorithm for ultrasonic elasticity imaging, Medical image analysis 13 (2009), 286-296.

[12] H. Rivaz, E. Boctor, P. Foroughi, R. Zellars, G. Fichtinger, and G. Hager, Ultrasound Elastography: A Dynamic Programming Approach, Medical Imaging, IEEE Transactions on 27 (2008), 1373-1377.

[13] J. Meunier and M. Bertrand, Ultrasonic texture motion analysis: theory and simulation, Medical Imaging, IEEE Transactions on 14 (1995), 293-300.

[14] J.A. Jensen and S.I. Nikolov, Fast simulation of ultrasound images, Proceedings of IEEE Ultrasonics Symposium, 2000 (2000), 1721-1724.

[15] I. Cespedes and J. Ophir, Reduction of image noise in elastography, Ultrasonic Imaging 15 (1993), 89-102.

[16] S. Srinivasan, R. Righetti, and J. Ophir, Trade-offs between the axial resolution and the signal-to-noise ratio in elastography, Ultrasound in medicine \& biology 29 (2003), 847-866. 